\title{
Orbital Specific Chirality and Homochiral Self-Assembly of Achiral Molecules Induced by Charge Transfer and Spontaneous Symmetry Breaking
}

\author{
A. Mugarza, ${ }^{1}$ N. Lorente, ${ }^{1}$ P. Ordejón, ${ }^{1}$ C. Krull, ${ }^{1}$ S. Stepanow, ${ }^{1}$ M.-L. Bocquet, ${ }^{2}$ J. Fraxedas, ${ }^{1}$ \\ G. Ceballos, ${ }^{1}$ and P. Gambardella ${ }^{1,3}$ \\ ${ }^{1}$ Centre d'Investigació en Nanociència i Nanotecnologia (ICN-CSIC), UAB Campus, E-08193 Bellaterra, Spain \\ ${ }^{2}$ Laboratoire de Chimie, UMR 5532, Ecole Normale Supérieure, F-69007 Lyon, France \\ ${ }^{3}$ Institució Catalana de Recerca i Estudis Avançats (ICREA), E-08100 Barcelona, Spain
}

(Received 26 May 2009; revised manuscript received 16 July 2010; published 10 September 2010)

\begin{abstract}
We study the electronic mechanisms underlying the induction and propagation of chirality in achiral molecules deposited on surfaces. Combined scanning tunneling microscopy and $a b$ initio electronic structure calculations of Cu-phthalocyanines adsorbed on $\operatorname{Ag}(100)$ reveal the formation of chiral molecular orbitals in structurally undistorted molecules. This effect shows that chirality can be manifest exclusively at the electronic level due to asymmetric charge transfer between molecules and substrate. Single molecule chirality correlates with attractive van der Waals interactions, leading to the propagation of chirality at the supramolecular level. Ostwald ripening provides an efficient pathway for complete symmetry breaking and self-assembly of homochiral supramolecular layers.
\end{abstract}

PACS numbers: 64.75.Yz, 68.37.Ef, 68.43.-h, 81.16.Dn

Chirality plays a fundamental role in molecular recognition processes, providing selectivity to many liferegulating chemical reactions. Chiral molecules exist in two nonsuperposable mirror-image forms, called enantiomers, that display dramatically different activity in important classes of compounds such as pharmaceuticals and crop-protection chemicals [1]. To improve the production of enantiomerically pure compounds, chirally modified metal surfaces have been intensively studied in the last two decades as heterogeneous catalysts $[2,3]$. Recently, chiral surfaces have assumed central importance to a diverse range of investigations, including the amplification of nonlinear optical properties [4] and the asymmetric scattering of spin-polarized electrons $[5,6]$. Common to these fields, is the problem of how to confer chiral properties to otherwise achiral metal substrates.

A particularly efficient way of bestowing chirality to surfaces relies on the deposition of organic molecular films $[7,8]$. In such systems, chirality can be expressed at different levels: local (single molecule) [9], organizational (supramolecular) $[10,11]$, or both $[2,12]$. It is well known that the adsorption of chiral molecules leads to the expression of both types of chirality. However, naturally occurring achiral molecules may also become chiral upon deposition on single-crystal surfaces, adding handedness to a vast library of chemical functions. This has been observed for specific (prochiral) molecular geometries owing to confinement in two dimensions $[13,14]$ as well as molecules that undergo conformational changes upon adsorption $[15,16]$. Highly symmetric molecules locked to a substrate with dissimilar point-group symmetry have also been shown to develop chiral ordering through asymmetric intermolecular interactions $[17,18]$. Although a significant effort has been directed towards the structural character- ization of adsorbed molecules and supramolecular domains in relation to chirality, little is known about their electronic properties and substrate-molecule interactions that ultimately lead to chirally modified surfaces.

In this Letter, we address the mechanisms of chiral induction and propagation of achiral molecules deposited on a metallic substrate. We present the case of squareplanar $\mathrm{Cu}$-phthalocyanines (CuPc) adsorbed on $\mathrm{Ag}(100)$ as a model system where the individual symmetry of adsorbate and substrate does not necessarily lead to chirality. Scanning tunneling microscopy (STM) measurements and density functional theory (DFT) calculations reveal the formation of chiral molecular orbitals in a structurally undistorted molecule, showing that substrate-induced enantiomerism can be of purely electronic nature and escape structural investigations. Attractive intermolecular interactions lead to the formation of supramolecular clusters with organizational chirality correlated to the handedness of the individual molecules. We further show that spontaneous symmetry breaking occurs thanks to the amplification of small inequalities in the initial distribution of supramolecular enantiomers and reversible single molecule chirality, leading to the self-assembly of electronically and structurally enantiopure layers over mesoscopic dimensions.

Figure 1(a) shows the adsorption geometry of $\mathrm{CuPc}$ monomers on $\operatorname{Ag}(100)$, deposited in ultrahigh vacuum by organic molecular beam epitaxy with the sample at room temperature. STM measurements were carried out in situ at $5 \mathrm{~K}$. Similarly to previous investigations on low-index metal surfaces [19,20], CuPc molecules adsorb parallel to the substrate plane. We observe two different azimuthal orientations corresponding to molecules rotated by $\pm 30^{\circ}$ $\left( \pm 2^{\circ}\right)$ relative to the [011] surface direction, with the 


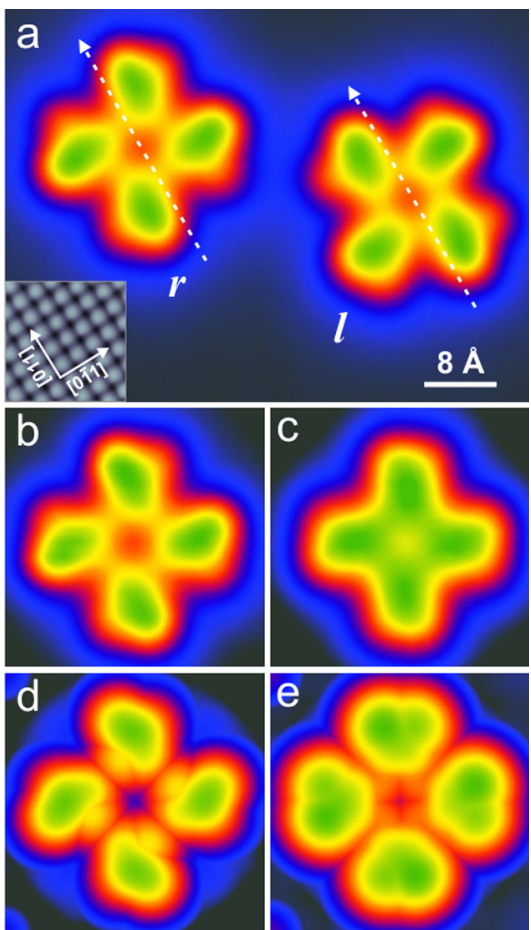

FIG. 1 (color online). STM images of $\mathrm{CuPc}$ adsorbed on $\operatorname{Ag}(100)$. (a) Molecules rotated by $+/-30^{\circ}$ with respect to the [011] crystallographic direction (inset) show $r / l$ chirality at bias voltage $V_{b}=-0.3 \mathrm{~V}$. (b) STM image of $r$-CuPc obtained at $V_{b}=-1 \mathrm{~V}$ and (c) $V_{b}=+0.3 \mathrm{~V}$ compared to the corresponding (d) and (e) STM images calculated using DFT.

$\mathrm{Cu}$ ion on $\mathrm{Ag}$ hollow sites in both cases. STM images reveal strong and opposite chiral contrast for the two molecular orientations, denoted as $r$ and $l$, respectively, which are mirror-symmetric with respect to the [011] axis. Enantiomorphism in a symmetry-matched moleculesubstrate system represents a limiting case where pointgroup symmetry constraints alone cannot explain chiral induction $[11,17,18]$. Remarkably, chiral contrast in the STM images is strong at negative bias and progressively disappears at positive bias voltage, as shown in Figs. 1(b) and 1(c). This behavior suggests that chirality in this system is mainly of electronic origin, i.e., not related to the molecule conformation.

To unravel the complex electronic interactions that give rise to enantiomorphism in $\mathrm{CuPc}$, we have performed an extensive set of $a b$ initio calculations using the VASP implementation of DFT in the projected augmented plane wave scheme and the local density approximation (LDA) [22]. Results obtained with the generalized gradients (GGA) approximation differ only quantitatively, with LDA producing a stronger molecule-surface interaction that leads to a shorter adsorption distance and larger charge transfer. The calculated slab included $5 \mathrm{Ag}$ atomic layers intercalated by 8 vacuum layers, and a $7 \times 7$ lateral supercell, relaxed until forces were smaller than $0.04 \mathrm{eV} / \AA$ [23]. In agreement with STM, we find that the preferred location of the $\mathrm{Cu}$ ion is the $\mathrm{Ag}$ hollow site, with a $0.14 \mathrm{eV}$ energy gain over the next stable configuration. With respect to top sites, the $\mathrm{Cu}$-surface distance decreases from 2.7 to $2.4 \AA$, leading to larger charge transfer from the substrate and stronger stabilization of the molecule. The minimum-energy azimuthal orientation corresponds to a $32^{\circ}$ deviation of the $\mathrm{Cu}$-ligand axis from the [011] direction of the surface. The driving force underlying such a rotation is the $\mathrm{N}-\mathrm{Ag}$ bond that forms between the $s p^{2}$ orbitals localized on the $a z a-\mathrm{N}$ atoms and the nearest $\mathrm{Ag}$ atoms of the top layer. In order to minimize the $\mathrm{N}-\mathrm{Ag}$ distance, the molecule rotates as shown in Figs. 2(a) and 2(b).

The optimized CuPc configuration shows a remarkable net transfer of 0.85 electrons into the molecule, obtained using the Bader charge analysis [24], distributed mainly into the doubly-degenerate lowest unoccupied molecular orbital (LUMO) and, to a lesser extent, into the unoccupied counterpart of the singly occupied molecular orbital (SOMO). This is reflected in the projected density of states (PDOS) shown in Fig. 2(c), where both LUMO and minority spin SOMO resonances partially cross the Fermi level, shifting down by about 0.5 and $0.2 \mathrm{eV}$, respectively, with respect to LDA calculations of gas-phase $\mathrm{CuPc}$ [25]. The differential charge density of the $\mathrm{CuPc} / \mathrm{Ag}(100)$ complex relative to $\mathrm{CuPc}$ and $\mathrm{Ag}(100)$ is reported in Figs. 2(a) and 2(b). The excess charge partly localizes on the $\mathrm{Cu}$ atom, as expected from the SOMO downshift, and, more importantly, on the $\mathrm{N}$ atoms and benzyne groups, reproducing the charge contour of the frontier orbitals [25]. Specifically, as the $s p^{2}$ states of the $a z a-\mathrm{N}$ atoms have considerable weight in the LUMO, charge transfer largely involves the $\mathrm{N}-\mathrm{Ag}$ bonds. These results show that the
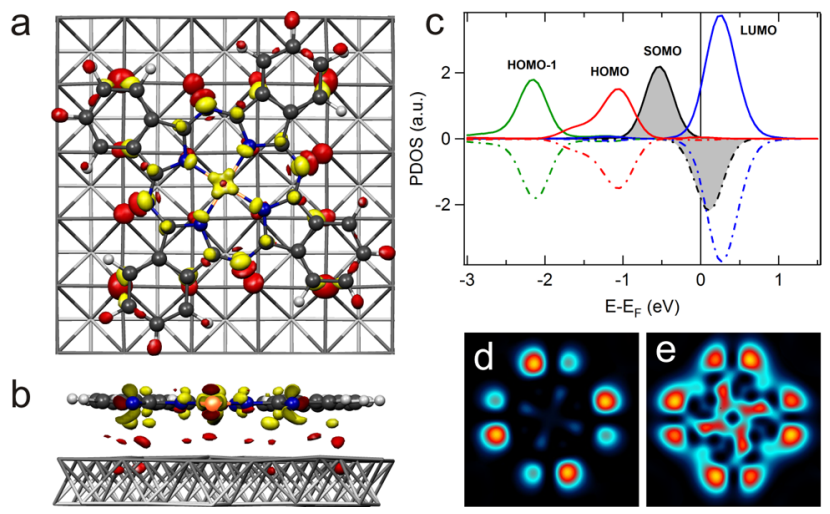

FIG. 2 (color online). (a) Top- and (b) diagonal side view of the differential charge density induced by molecular bonding to the substrate. The contour value is $\pm 3.8 \times 10^{-3} \mathrm{e} / \AA^{3}$. The excess (depletion) of charge is shown in yellow (red). (c) Calculated DOS of $r-\mathrm{CuPc} / \mathrm{Ag}(100)$ projected onto the unperturbed $\mathrm{CuPc}$ molecular orbitals. Positive (negative) units refer to spin up (down) states; a Gaussian broadening of $0.25 \mathrm{eV}$ has been employed. (d),(e) Theoretical conductance maps at the peak position of the (d) HOMO and (e) LUMO. 
adsorption of $\mathrm{CuPc}$ on $\mathrm{Ag}(100)$ implies a considerable degree of chemical bonding and that the molecular configuration is determined by $\mathrm{N}$-specific interactions with the substrate. Importantly, the calculations disclose no significant distortion of the molecule, which remains planar as depicted in Fig. 2(b), showing no geometrical chirality due to adsorption.

The above findings explain the achiral topography of CuPc observed by STM at positive bias [Fig. 1(c)], and allow us to elucidate the electronic origin of chirality in the adsorbed molecules observed at negative bias [Figs. 1(a) and 1(b)]. The main source of chirality is the distortion of the highest occupied molecular orbital (HOMO) caused by direct interaction of the benzyne groups with the substrate atoms. This can be best observed by plotting the conductance maps obtained from the simulations at the HOMO/ LUMO resonances [Figs. 2(d) and 2(e)], where the asymmetry between the two lobes located at the benzyne ring position is significantly more pronounced in the HOMO compared to the LUMO. Considering that the SOMO gives a negligible contribution to the tunneling current due to its $d_{x^{2}-y 2}$ character $[20,21]$, the chiral contrast observed in the experimental and simulated STM images of Fig. 1, tuned to the HOMO/LUMO energy levels at -1.0 and $0.3 \mathrm{eV}$, respectively, reflect the different asymmetry of each orbital. The point chirality of CuPc molecules is therefore attributed to an electronic effect, which induces orbitalselective enantiomorphism of the molecular electron states in a well-defined energy range near the Fermi level.

A key question then is whether the chirality of the $\mathrm{CuPc}$ electron states can be transferred to extended twodimensional layers. Chirality in adsorbed molecular arrays requires stereospecific molecular interactions, which are usually provided through either hydrogen bonds $[13,14]$ or correlation of azimuthal molecular orientations with lateral van der Waals (VdW) forces [17,18]. Macrocyclic molecules such as phthalocyanines $[19,20,26]$ and porphyrins $[27,28]$ are known to assemble into ordered compact layers at surfaces, where spontaneous formation of organizational chiral domains occurs as a result of lattice-matching constraints and weak VdW attraction. In the absence of local enantiomorphism, however, the chirality expression is limited to the structure of the arrays. Further, there is no reason for one type of domain to prevail upon the other, and the coexistence of both type of organizational domains in the same molecular layer is observed [26-28]. The selfassembly of $\mathrm{CuPc}$ on $\mathrm{Ag}(100)$ significantly differs from such behavior, since the electronic chirality of each molecule is carried over to extended layers and correlated to organizational chirality. Moreover, spontaneous symmetry breaking occurs, leading to the formation of CuPc films with complete chiral purity over mesoscopic dimensions.

Figures 3(a) and 3(b) are representative of mirrorsymmetric enantiopure $\mathrm{CuPc}$ domains. We observe single handedness of the molecular arrangement for each enan-
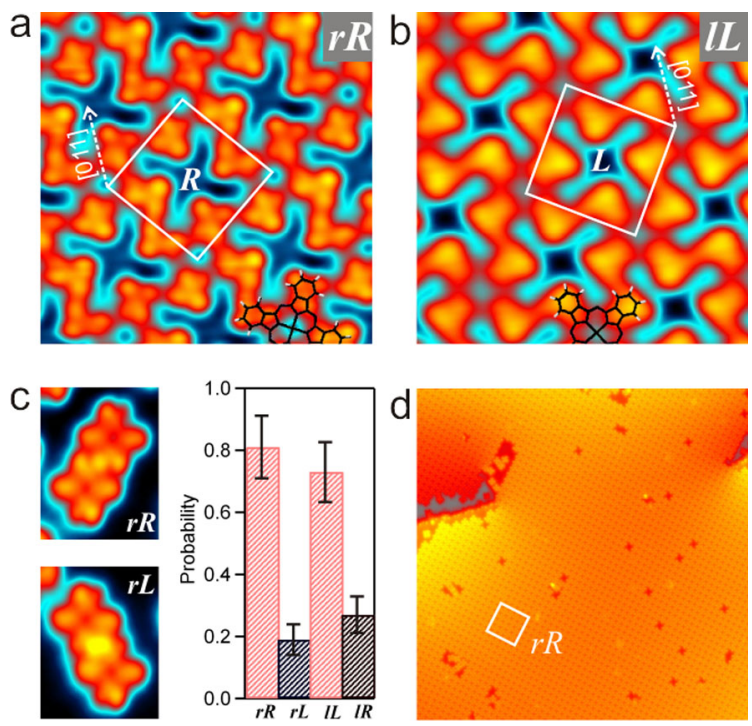

FIG. 3 (color online). Chiral self-assembly of CuPc. (a) $r R$ and (b) $l L$ homochiral domains recorded with $V_{b}=+0.52 \mathrm{~V}$ and $-0.10 \mathrm{~V}$ respectively. (c) $r R$ and $r L$ dimers shown together with the relative probability of each configuration (d) Homochiral $r R$ domain extending over a terrace and crossing a screw dislocation. Image size $77.3 \times 77.3 \mathrm{~nm}$.

tiomer, with the two domains corresponding to commensurate superlattices with $5 \times 5$ periodicity, rotated by $\pm 37^{\circ}$ with respect to the [011] direction. The superlattice chirality is designated as $R(L)$ for the $5 \times 5 R-(+) 37^{\circ}$ structure. The univocal relation between molecular and array chirality results in the preferential assembly of $r R$ and $l L$ domains, although a similar hollow site adsorption and CuPc close-packing fraction would be obtained for $r L$ and $l R$ configurations having $5 \times 5 R 0^{\circ}$ periodicity [23]. Figure 3(c) shows that this chiral propagation process is already active between isolated CuPc enantiomers, as the relative abundance of $r R / r L$, and $l L / l R$ dimers in the earliest stages of aggregation is strongly asymmetric. To understand the mechanism of chiral recognition, we carried out calculations of both $l L$ and $l R$ phases $(r R$ and $r L$ being equivalent by symmetry) using SIESTA [29], and a new DFT functional explicitly designed to include VdW interactions $[30,31]$. The calculations show that the orientation of $\mathrm{CuPc}$ with respect to the substrate is the same for the two phases, so the structure of the monolayer is mainly determined by the CuPc-Ag interaction. However, the distances between adjacent phenyl groups in the $l L$ phase are slightly smaller compared to $l R$, leading to a stabilization of the former due to attractive $\mathrm{VdW}$ interactions. The total energy difference between the two phases is of the order of $40 \mathrm{meV}$, which is reduced below the relative accuracy of the calculations $(\sim 10 \mathrm{meV})$ by removing the nonlocal VdW correlation [23]. We note that substratemediated enantiospecific interactions [32] may also lead to chiral recognition. Indeed, we find that the charge transfer between $\mathrm{Ag}$ and $\mathrm{CuPc}$ induces chiral asymmetry to the 
substrate metal states in the proximity of each molecule, but the resulting electrostatic potential energy difference between the $l L$ and $l R$ dimer configurations cancels out after integration over the whole area occupied by neighbor molecules [23].

Our data show that a racemic mixture of $r R$ and $l L$ domains is present on each terrace in the initial stages of growth (Fig. S4 in Ref. [23]), consistently with the fact that they are energetically equivalent. As the $\mathrm{CuPc}$ coverage approaches one monolayer; however, we observe that the symmetric population spontaneously breaks into a single homochiral phase, the extension of which is limited only by substrate steps [Fig. 3(d)]. This phenomenon is quite unusual since spontaneous organizational chiral resolution is not reported for similar systems [26-28]. Even for crystallization reactions in solution, achieving complete chiral purity is very rare when both enantiomers are present since the beginning [33]. However, mechanisms that enhance any initial imbalance in chirality and catalyze the production of one enantiomer while suppressing its mirrorimage are important as they provide clues to spontaneous chiral symmetry breaking in nature. In the present case, chiral resolution occurs through two-dimensional Ostwald ripening, which allows for the exchange of molecules between supramolecular clusters of opposite handedness during the nucleation and growth process at room temperature [23]. Note that complete chiral purity can be reached only because both $r R$ and $l L$ domains can independently feed the growth of the majority phase. This is due to the intrinsic achiral nature of the molecules, which allows for switching of the surface-induced point-group chirality during molecular diffusion.

In conclusion, we have shown that surface-induced chirality can arise as a purely electronic effect irrespective of point-group symmetry arguments and propagate at the organizational level through chiral recognition and spontaneous symmetry breaking. STM and DFT calculations reveal that $\mathrm{CuPc}$ adsorbed on $\operatorname{Ag}(100)$ display orbitalspecific electronic chirality caused by molecule-substrate asymmetric charge transfer, while the molecular conformation remains achiral. The electronic chirality of individual molecules is retained in extended layers and univocally transferred at the organizational level through lateral VdW interactions. With increasing molecular density, an equal mixture of supramolecular domains of opposite handedness evolves into mesoscopic homochiral $\mathrm{CuPc}$ layers due to spontaneous chiral symmetry breaking during selfassembly. The ability to grow enantiopure supramolecular layers and tune the chirality of distinct molecular orbitals may lead to novel ways to control the electronic properties and optical response of metal surfaces.

We acknowledge financial support from the Spanish Ministerio de Ciencia e Innovación (FIS2006-12117-
C04-01, FIS2009-12721-C04-01 and MAT2007-62341) and the European Research Council (StG 203239). N.L. also thanks the Agence Nationale de la Recherche (JCJC06-148337) for partial funding, the Centre de Calcul Midi-Pyrénnées, and the Barcelona Supercomputing Center.

[1] H.-J. Federsel, Nat. Rev. Drug Discov. 4, 685 (2005).

[2] M. O. Lorenzo et al., Nature (London) 404, 376 (2000).

[3] G. J. Hutchings, Annu. Rev. Mater. Res. 35, 143 (2005).

[4] T. Verbiest et al., Science 282, 913 (1998).

[5] R. A. Rosenberg, M. A. Abu Haija, and P. J. Ryan, Phys. Rev. Lett. 101, 178301 (2008).

[6] K. Ray et al., Science 283, 814 (1999).

[7] S. M. Barlow and R. Raval, Surf. Sci. Rep. 50, 201 (2003).

[8] K.-H. Ernst, Top. Curr. Chem. 265, 209 (2006).

[9] G. P. Lopinski et al., Nature (London) 392, 909 (1998).

[10] F. Charra and J. Cousty, Phys. Rev. Lett. 80, 1682 (1998).

[11] M. Parschau et al., Angew. Chem., Int. Ed. 46, 8258 (2007).

[12] R. Fasel, M. Parschau, and K.-H. Ernst, Nature (London) 439, 449 (2006).

[13] M. Böhringer et al., Phys. Rev. Lett. 83, 324 (1999).

[14] J. Weckesser et al., Phys. Rev. Lett. 87, 096101 (2001).

[15] Q. Chen, D. Frankel, and N. Richardson, Surf. Sci. 497, 37 (2002).

[16] S. Weigelt et al., Nature Mater. 5, 112 (2006).

[17] M. Schock et al., J. Phys. Chem. B 110, 12835 (2006).

[18] N. V. Richardson, New J. Phys. 9, 395 (2007).

[19] P. H. Lippel et al., Phys. Rev. Lett. 62, 171 (1989).

[20] X. Lu et al., J. Am. Chem. Soc. 118, 7197 (1996).

[21] S. Stepanow, A. Mugarza, G. Ceballos, P. Moras, J. C. Cezar, C. Carbone, and P. Gambardella, Phys. Rev. B 82, 014405 (2010)

[22] G. Kresse and D. Joubert, Phys. Rev. B 59, 1758 (1999).

[23] See supplementary material at http://link.aps.org/ supplemental/10.1103/PhysRevLett.105.115702.

[24] R. F. W. Bader, W. H. Henneker, and P. E. Cade, J. Chem. Phys. 46, 3341 (1967).

[25] A. Calzolari, A. Ferretti, and M. B. Nardelli, Nanotechnology 18, 424013 (2007).

[26] Z. H. Cheng et al., J. Phys. Chem. C 111, 2656 (2007).

[27] W. Auwärter et al., J. Chem. Phys. 124, 194708 (2006).

[28] D. Ecija et al., J. Phys. Chem. C 112, 8988 (2008).

[29] J. M. Soler et al., J. Phys. Condens. Matter 14, 2745 (2002).

[30] G. Román-Peréz and J. M. Soler, Phys. Rev. Lett. 103, 096102 (2009).

[31] M. Dion, H. Rydberg, E. Schröder, D. C. Langreth, and B. I. Lundqvist, Phys. Rev. Lett. 92, 246401 (2004).

[32] S. Blankenburg and W. G. Schmidt, Phys. Rev. Lett. 99, 196107 (2007).

[33] C. Viedma, Phys. Rev. Lett. 94, 065504 (2005). 


\title{
Supplemental Material
}

\section{Orbital specific chirality and homochiral self-assembly of achiral molecules induced by charge transfer and spontaneous symmetry breaking}

\author{
A. Mugarza ${ }^{1}$, N. Lorente ${ }^{1}$, P. Ordejón ${ }^{1}$, C. Krull ${ }^{1}$, S. Stepanow ${ }^{1}$, \\ M.-L. Bocquet ${ }^{2}$, J. Fraxedas ${ }^{1}$, G. Ceballos ${ }^{1}$, and P. Gambardella ${ }^{1,3}$ \\ ${ }^{1}$ Centre d'Investigació en Nanociéncia i Nanotecnologia (ICN-CSIC), \\ UAB Campus, E-08193 Bellaterra, Spain \\ ${ }^{2}$ Laboratoire de Chimie, UMR 5532, Ecole Normale Supérieure, \\ F-69007 Lyon, France \\ ${ }^{3}$ Institució Catalana de Recerca i Estudis Avançats (ICREA), \\ E-08100 Barcelona, Spain
}

\section{Chiral structure of the supramolecular domains}

STM images of CuPc adsorbed on $\mathrm{Ag}(100)$ reveal different levels of chiral expression. At the single molecule level, point chirality induced by charge transfer gives rise to two molecular enantiomers, $r$ and $l$, which are energetically equivalent because they are mirror symmetric with respect to the surface primitive vectors $\{1 / \sqrt{ } 2[011], 1 / \sqrt{ } 2[0-11]\}$ In the following, to simplify the notation, we consider only the $l$ enantiomer and the related superlattices. The measured angle between molecular and surface symmetry axes slightly reduces from $-30^{\circ}$ to $-27^{\circ}$ in the superlattices (negative sign corresponds to counterclockwise, i.e. left, rotation). A similar reduction of the azimuthal angle is also observed in the relaxed structures obtained in the calculations.

At the organizational level, supramolecular chirality arises as the molecules selfassemble into mirror symmetric domains, designated as $R$ and $L$. The superlattice structure that we observe for the $l$ molecule, shown in Figure $S 1$, is the $I L$, which is commensurate with the surface with a $5 \times 5$ periodicity. In Wood's notation, the $I L$ superstructure is denoted as $5 \times 5 \mathrm{R} 37^{\circ}$, whereas in matrix notation, using the basis of the surface unit cell, the same structure is expressed as

$$
M_{I L}=\left(\begin{array}{cc}
4 & +3 \\
-3 & 4
\end{array}\right)
$$

The $I L$ superlattice thus consists of a square unit cell, rotated by $-26.1^{\circ}$ with respect to the molecular axis direction, i.e., by $-27^{\circ}-26.1^{\circ}=-53.1^{\circ}$ with respect to the surface primitive vectors, as shown in Figure S1. Due to the 4-fold symmetry of the surface this is equivalent to $\mathrm{a}-53.1^{\circ}+90^{\circ}=+36.9^{\circ}$ rotation, which defines the proper rotation angle in the reference frame of the surface. Due to the counterclockwise (left) rotation of the superlattice unit cell with respect to the molecular axis, we use the letter $L$ to denote its chirality. 


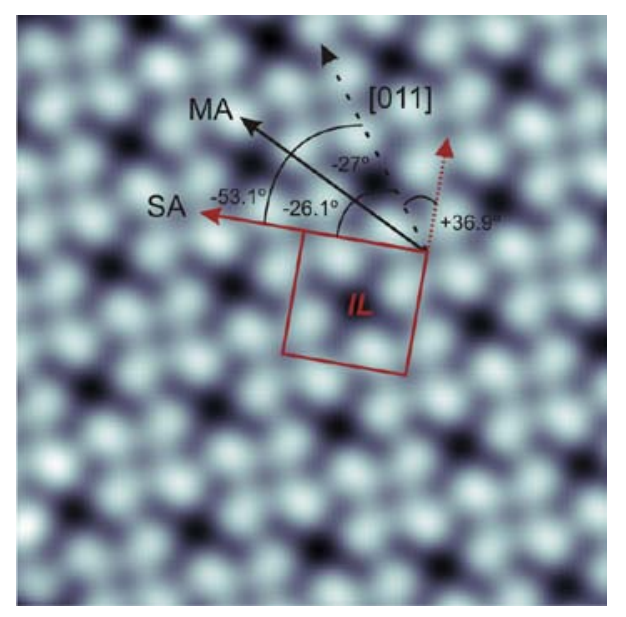

FIGURE S1. STM image showing the $I L$ superlattice configuration. The molecular axis (MA) and superlattice unit cell directions (SA) and angles with respect to the [011] surface direction are superposed.

The corresponding mirror symmetric superstructure would be rotated by $+26.1^{\circ}$ with respect to the molecular axis, leading to a unit cell closely aligned $\left(-27^{\circ}+26.1^{\circ}=-0.9^{\circ}\right)$ with respect to the primitive vectors of the $\mathrm{Ag}(100)$ surface. The equivalent superlattice matrix is

$$
M^{\prime}{ }_{I R}=\left(\begin{array}{cc}
5.001 & +0.087 \\
-0.087 & 5.001
\end{array}\right) .
$$

Evidently, this structure is not commensurate with the surface. As the molecule-substrate interaction dominates over intermolecular forces, the molecules accommodate to the surface lattice, forming a slightly modified $5 \times 5$ superstructure. The closest commensurate superlattice corresponds to a rotation of the unit cell by $+27^{\circ}$ with respect to the molecular axis, i.e., to a $5 \mathrm{x} 5 \mathrm{R} 0^{\circ}$ structure or

$$
M_{l R}=\left(\begin{array}{ll}
5 & 0 \\
0 & 5
\end{array}\right) .
$$

The superlattice structures corresponding to the $r$ molecule are the $5 \times 5 \mathrm{R}-37^{\circ}$ for $r R$ and $5 \mathrm{x} 5 \mathrm{R} 0^{\circ}$ for $r L$. Note that the latter coincides with $I R$, although the molecule is rotated in the opposite direction. The $I R$ and $r L$ configurations are observed by low energy electron diffraction (LEED) at room temperature, coexisting with $l L$ and $r R$ (Figure S2). As the mobility of the molecules decreases due to either a reduction of temperature or completion of the first CuPc layer, only the $l L$ and $r R$ phases survive. DFT calculations (see Sect. 2) show that the $I R$ monolayer has a higher total energy compared to $l L$ by about $40 \mathrm{meV}$, consistently with the observed coexistence of $I R / r L$ and $I L / r R$ at room temperature and preference for $l L / r R$ at low temperature. 


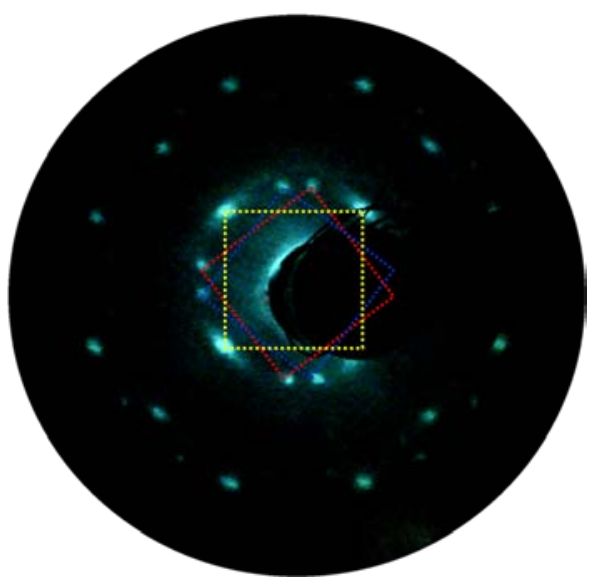

FIGURE S2. LEED pattern recorded at room temperature showing the coexistence of $r R$ (red), $I L$ (blue), and $r L / l R$ domains (yellow square).

\section{Ab-initio calculations of supramolecular CuPc chiral phases}

The calculations of the energetics of CuPc monolayers on the $\operatorname{Ag}(001)$ surface have been performed using the SIESTA code [1], with a double- $\zeta$ polarized basis-set specifically developed for noble metal surfaces [2]. The slabs used to simulate the surfaces included $5 \mathrm{Ag}$ atomic layers intercalated by 8 vacuum layers in the vertical direction. All the calculations were carried out using fully relaxed structures (until residual forces were smaller than 0.04 $\mathrm{eV} / \AA$ ), while keeping the three lower layers of the slab fixed to mimic the effect of the bulk material. All energies were well converged with respect to the k-point sampling and the real space mesh cutoff. The calculations (including the atomic relaxations) were performed using two different DFT functionals:

1) The LDA functional of Perdew and Zunger [3]. This functional gives a good account of chemically bonded systems, but it is questionable for the description of weakly bonded systems. In particular, LDA fails completely in describing the dispersion interaction between non-overlapping fragments, i.e. asymptotic Van der Waals (VdW) interactions. For VdW complexes, where the equilibrium geometry is in the intermediate overlapping regime, LDA often produces distances and binding energies in reasonable agreement with experimental ones due to an exchange residue [J. Harris, Phys. Rev. B 31, 1770, 1985], even if long range correlation terms are not explicitly included in this approximation.

2) A new DFT functional (DSRLL) was designed to include explicitly the VdW interactions, developed recently by the groups of D. Langreth and B. Lundqvist [4-6]. We have used the implementation of this functional developed by G. Román-Pérez and J. M. Soler [7] that is available in SIESTA. This functional is thought to provide a better description of the molecule-surface and molecule-molecule interactions compared to LDA. 
We have performed calculations for monolayers of CuPc on the Ag(100) surface adopting the $I L$ and $I R$ configurations defined in Sect. 1, obtaining the relaxed structures and energies for both configurations. The results of our calculations show that:

(i) The structure of the monolayer is mainly determined by the interaction between each individual molecule and the $\mathrm{Ag}(100)$ surface. The angle between the molecular symmetry axes and the substrate crystallographic directions in the relaxed structures is essentially the same for both structures (see surface axis directions in Figure S3), indicating that intermolecular interactions in the monolayer are less important than molecule-surface interactions. This is the case for both of the DFT functionals used.

(ii) The contact distances between neighbouring molecules are different for the $I L$ and $I R$ structures. This is due to the fact that the two assemblies display the same angle between molecule and substrate axes and the same superlattice periodicity but different unit cell vectors. As a consecuence, neighbours appear in different directions. The intermolecular contacts are shorter for the monolayer in the $I L$ phase, as shown in Figure S3, because the relative orientation between neighbour molecules is such that two phenyl groups are closer in this case.

(iii) The total energies computed for the relaxed structures show that the $l L$ phase is more stable by a few tenths of eV (see Table I for details), both for LDA and the VdW DRSLL functionals.

The origin of the energy difference between the two structures, and the preference towards the $I L$ phase observed in the experiment, is attributed to the stronger VdW (attractive) interaction between neighbour molecules that exist in this phase, due to the slightly closer
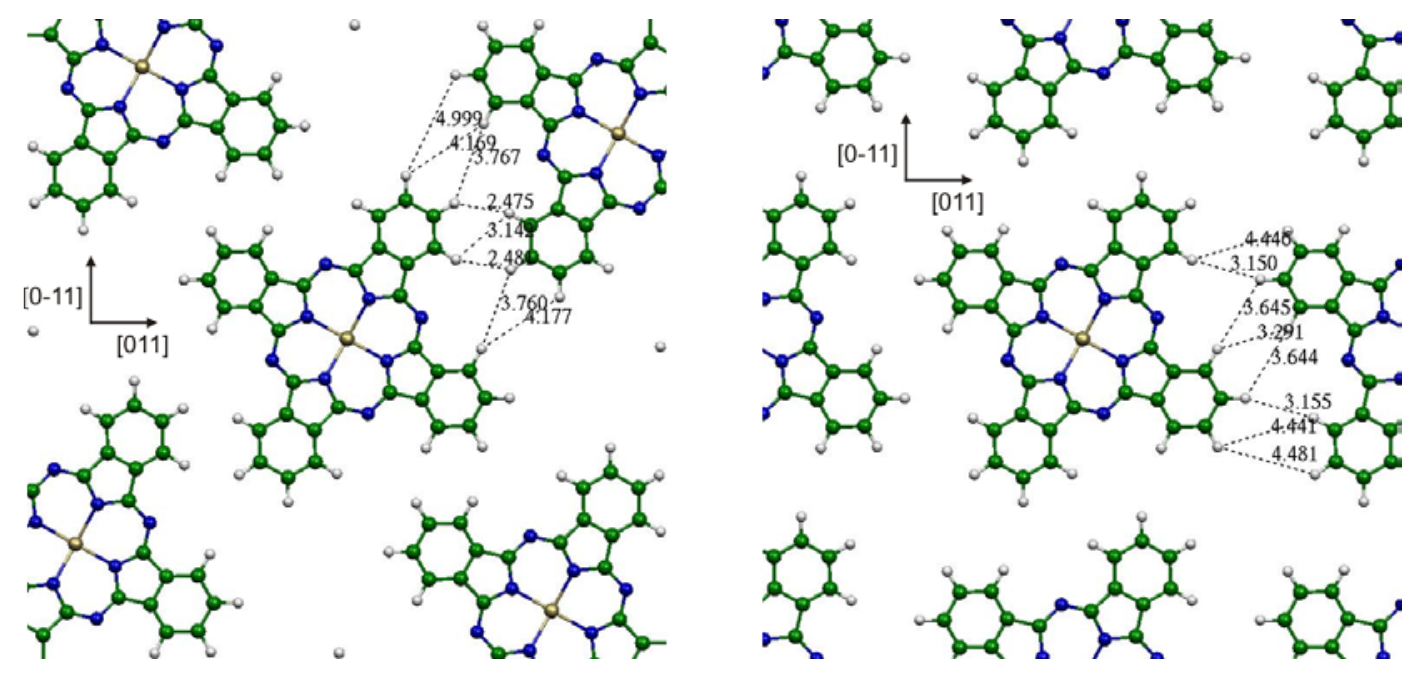

FIGURE S3. Scheme of the structure of the $I L$ (left) and $I R$ (right) monolayers (Ag atoms are not shown for the sake of clarity), indicating the contact distances between neighbour molecules. Results obtained with the DSRLL functional. 
distances between the molecular endgroups. To show this, we have performed two separate calculations.

First, we have computed the energy of both $I L$ and $I R$ phases, but without including the VdW (non-local correlation) terms in the DRSLL functional. The results are shown in the last column of Table I. We see that the energy difference between the two phases is now very small, indicating that: (i) the energy of interaction between the molecule and the surface is similar in both structures, due to the similarity in the angles discussed previously; (ii) the molecules are sufficiently far so that the repulsive part of the interactions (which is present in the calculation) is negligible, and (iii) the effect of the interaction between molecules through the electronic states of the metal does not play an important role in the energy difference between the phases. Substrate mediated interactions are discussed in Sect. 3.

A second calculation allows us to conclude that the main source of the energy difference between both phases is the VdW interaction. We present in Table I the energy difference computed taking into account only the atoms of the adsorbed molecular monolayer, removing the metallic substrate but using the relaxed coordinates obtained for the whole system. We find that again the $I L$ phase has a lower energy compared to $I R$ and that most of the energy difference disappears when the VdW terms are excluded from the calculation.

It is interesting to note that the energy difference between both phases is smaller for the monolayer over the metallic surface than for the free monolayer, by almost a factor of two. This may be due to the fact that the metallic surface screens the VdW interactions [8], thus reducing the effect on the total energies and the energy difference. We also note that the LDA results show the same tendencies than those obtained with the DSRLL functional, although with larger energy differences. This is also common, since LDA tends to overestimate the binding at short distances in VdW complexes.

\begin{tabular}{l|lll}
\hline & LDA & DSRLL & $\begin{array}{l}\text { DSRLL } \\
\text { without VdW part }\end{array}$ \\
\hline$\Delta \mathrm{E}$ (ML on Ag(001)) & -0.069 & -0.041 & 0.003 \\
$\Delta \mathrm{E}$ (free ML) & -0.094 & -0.074 & 0.016 \\
\hline
\end{tabular}

TABLE I. Energy differences between the $I L$ and the $I R$ structures $\left(\Delta \mathrm{E}=E_{l L}-E_{l R}\right.$, in $\left.\mathrm{eV}\right)$, computed with the different DFT functionals. Negative values indicate a preference for the $I L$ phase. 


\section{Role of substrate-mediated molecular interactions}

The large charge transfer between molecules and substrate is found to imprint chiral asymmetry not only in the molecular orbitals but also in the substrate metal states. To further investigate this point, we have modeled the adsorption of individual CuPc molecules using SIESTA and a 15x15 supercell to avoid possible periodic boundary effects. The analysis of the induced charge on the Ag atoms that surround the molecule reveals a chiral perturbation of the metal states and a consequent distortion of the electrostatic potential around the molecule, shown in Figure S4. Previous theoretical investigations have shown this to occur for molecules with proper gas-phase chiral conformation, i.e., tartaric acid adsorbed on $\mathrm{Ni}(100)$ [9] and phenylglicine on $\mathrm{Cu}(110)$ [10]. It has also been proposed that substrate mediated interactions may favor chiral recognition due to long-range Coulomb forces combined with adsorption site constraints [10]. These findings suggest that substratemediated enantiospecific interaction may play a role in the assembly of chiral domains. However, the total energy calculations reported in Sect. 2, in particular those in the absence of substrate, indicate that this is not the case for CuPc on $\mathrm{Ag}(100)$. To understand why it is so, we integrated the electrostatic potential induced by one molecule at the position that would be occupied by a second molecule in the $I L$ and $I R$ adsorption sites. The electrostatic interaction energy of one molecule due to the presence of the other one is

$$
E_{e l}=\int \rho_{t o t}(\mathbf{r}) \cdot V_{H}(\mathbf{r}) d \mathbf{r}
$$

where, $\rho_{\text {tot }}$ represents the total charge density of the second molecule (sum of electronic and nuclear charges), $\mathrm{V}_{\mathrm{H}}$ is the electrostatic potential induced by the first molecule, and $\mathbf{r}=(x, y, z)$ the spatial coordinate. Note that, for such large molecules, a full self-consistent calculation where the two molecules are included in the computation of the potential is at present prohibited by the supercell size limitations. We find that, even though the electrostatic potential is anisotropic up to a distance of about $15 \AA$ from the $\mathrm{Cu}$ ion, larger than the $\mathrm{Cu}-$ first benzyne ring neighbor (13 $\AA$ ), the energy difference $\Delta E_{e l}=E_{e l}(l R)-E_{e l}(l L)=3.5 \mathrm{meV}$
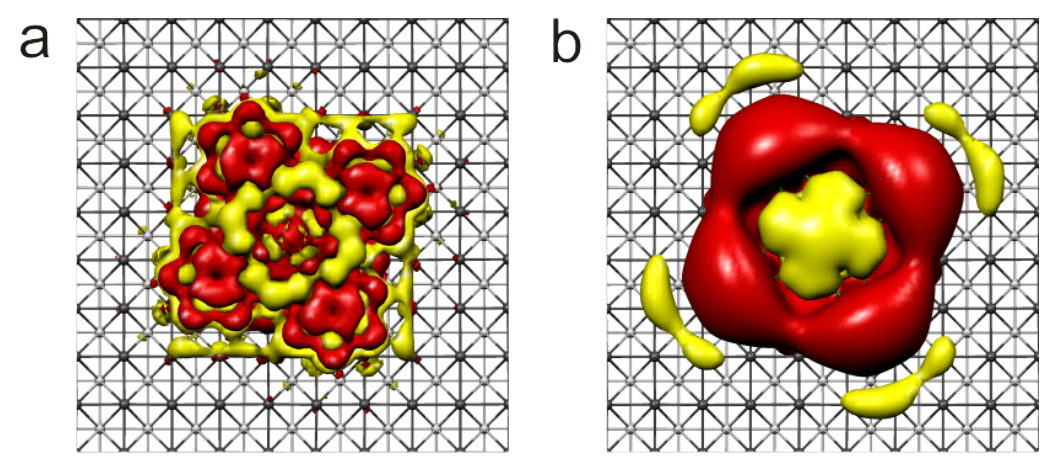

FIGURE S4. Extended differential electron density (a) and electrostatic potential (b) of an l-CuPc molecule adsorbed on a 15x15 supercell (only partially shown). The contours correspond to values of $\pm 7 \times 10^{-4} \mathrm{e} / \AA^{3}$ and $\pm 0.07 \mathrm{eV}$ for the density and potential, respectively. Yellow (red) indicates positive (negative) values. 
is negligible, after integration over the region occupied by the second molecule. $\Delta E_{e l}$ turns out to be about one order of magnitude smaller compared to the substrate-induced interaction estimated between opposite phenylglicine enantiomers and adenine on $\mathrm{Cu}(110)$ [10]. These molecules, however, have a much smaller surface footprint relative to Pc, suggesting that the effect of substrate-mediated interactions between enantiomers depend not only on charge transfer but also on the size of the adsorbates.

\section{Spontaneous chiral symmetry breaking during self-assembly}

The STM images displayed in Figure S5 illustrate the evolution of organizational chirality during the self-assembly process. The mechanisms behind each step are schematically illustrated in Figure S6. At low coverage, CuPc dimers have all possible bonding configurations, i.e., $r R, r L, l L, l R$, and even $r$ - $l$ (Figure $S 5$ a and b), although the population of each combination differs, reflecting its relative energy stability. In dimers, the energy difference between these configurations is due to a single bond and is not large enough to suppress the formation of metastable structures. Such metastable configurations, however, already disappear for tetramers and only a racemic mixture of $r R$ and $l L$ clusters is observed for larger assemblies (Figure S5 b). At coverages above approximately 0.5 ML, large islands of either $r R$ or $l L$ type develop on each terrace (Figure S5), growing at the expenses of the smaller ones. Near monolayer completion, each terrace consists of one enantiopure single-
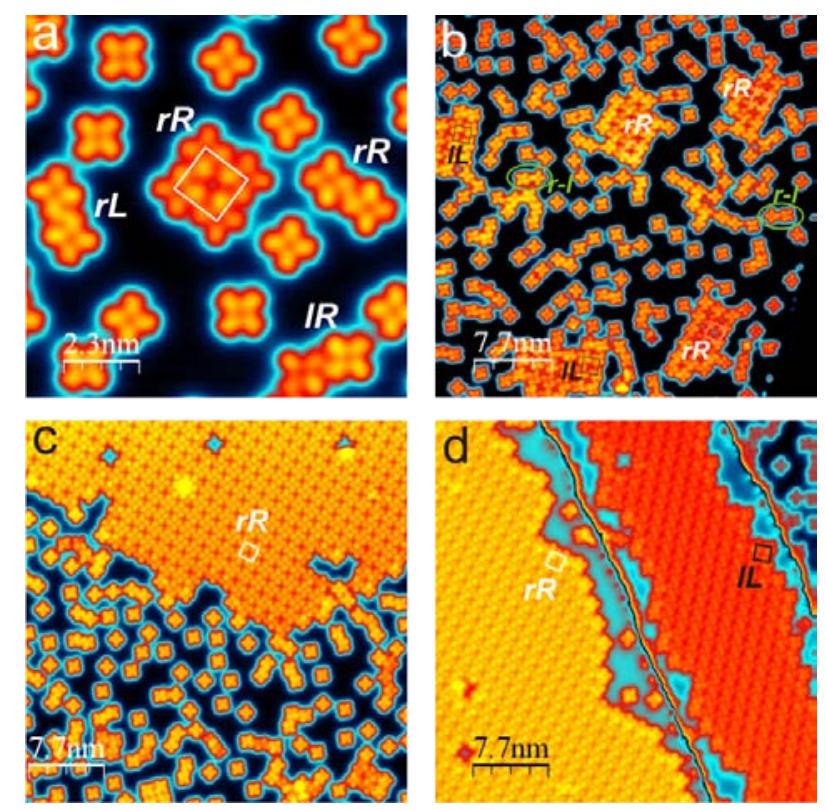

FIGURE S5: Coverage dependent evolution of organizational chirality. (a) At very low coverages, small clusters nucleate owing to attractive intermolecular interactions. Dimers are found in all possible bonding configurations. (b) At intermediate coverage, chiral recognition occurs for larger clusters, leading to a racemic mixture of either $r R$ or $l L$ structures. (c) Ostwald ripening favors the growth of one type of domain, leading to spontaneous symmetry breaking. (d) At monolayer completion, chiral purity is achieved on each terrace. 
domain phase (Figure S5 d). Such an Ostwald ripening process occurs thanks to the continuous exchange between molecular clusters and a two-dimensional "gas" of adsorbed molecules, similarly to what is known to take place for metal systems [11], which amplifies small initial fluctuations in the population of $r R$ and $l L$ enantiomers. Interestingly, the necessary condition that leads to chiral "purification" through Ostwald ripening is the ability of the molecules to switch chirality during diffusion by rotating over the surface plane. Such a recycling mechanism is allowed by the intrinsic achiral nature of the molecules employed in this study, but may be of general relevance for other systems where chirality is induced by adsorption. The low energy barrier for chirality switch is reflected also in tip induced diffusion experiments, where the molecule repeatedly switches the azimuthal adsorption angle during the lateral diffusion.

Mechanisms that enhance any random imbalance in chirality during crystallization are essential to understand chiral selection processes that take place in nature. Complete spontaneous symmetry breaking is a rare process for molecules in solution [12,13]. On surfaces, although the lower degree of freedom due to substrate-constrained growth has been successfully exploited to induce spontaneous resolution, a homogenously distributed racemic mixture of enantiomorphic domains is typically obtained. The crystallization of two dimensional enantiopure molecular layers has been seldom achieved and, similar to the threedimensional case, it has required an external driving force such as enantiomeric excess [12], a chiral modifier [13], chiral solvent [14], or magnetic field [15]. In the present work, symmetry breaking of chiral molecular layers occurs spontaneously, and is complete at the single terrace level. The extension of the homochiral layers is thus limited only by the morphology of the substrate, not by kinetic or thermodynamic effects.

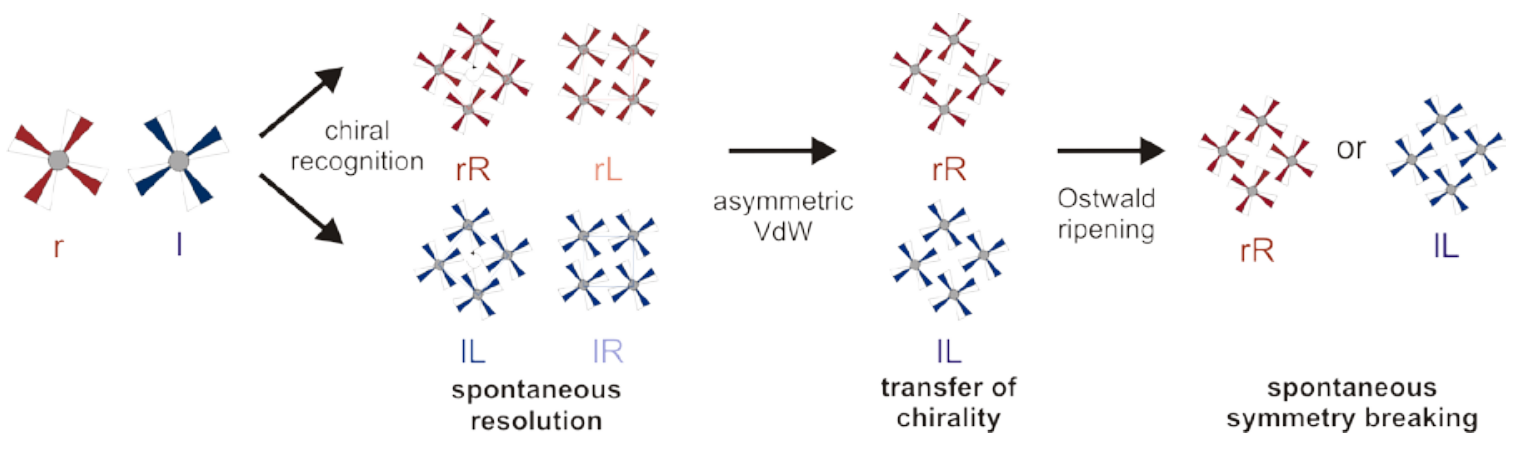

FIGURE S6. Schematics of the self-assembly mechanisms that induce homochirality on the CuPc/Ag(100) surface. The combination of adsorbate-substrate matching and VdW interactions is strong enough to induce chiral recognition (phase separation of $r$ and $l$ molecules), and transfer the chirality from the single-molecule level to the organizational level ( $r / l$ forming only $r R / l L$ islands). Ostwald ripening combined with reversible singlemolecule chirality favors the homochiral growth of the largest cluster at the expense of the smaller ones, leading to spontaneous $100 \%$ enantiomeric excess on each terrace. 


\section{References}

[1] J. M. Soler, E. Artacho, J. Gale, A. Garcia, J. Junquera, P. Ordejón, and D. SanchezPortal, J. Phys.: Condens. Matter 14, 2745 (2002).

[2] S. García-Gil, A. García, N. Lorente, and P. Ordejón, Phys. Rev. B 79, 075441 (2009).

[3] J. P. Perdue and A. Zunger, Phys. Rev. B 23, 5048 (1981).

[4] M. Dion, H. Rydberg, E. Schröder, D. C. Langreth, and B. I. Lundqvist, Phys. Rev. Lett. 92, 246401 (2004).

[5] T. Thonhauser, , V. R. Cooper, S. Li, A. Puzder, P. Hyldgaard, and D. C. Langreth, Phys. Rev. B 76, 125112 (2007).

[6] D. C. Langreth et al., J. Phys. Condens. Matter 21, 084203 (2009).

[7] G. Román-Pérez and J. M. Soler, Phys. Rev. Lett. 103, 096102 (2009).

[8] J. Mahanty, Phys. Rev. B 35, 4113 (1987).

[9] W. A. Hofer, V. Humblot, and R. Raval, Surf. Sci. 554, 141 (2004).

[10] S. Blankenburg and W. G. Schmidt, Phys. Rev. Lett. 99, 196107 (2007); Phys. Rev. B 78, 233411 (2008)

[11] H. Röder, E. Hahn, H. Brune, J.P. Bucher and K. Kern, Nature 366, 141 (1993).

[12] C. Viedma, Phys. Rev. Lett. 94, 065504 (2005).

[13] N. Petit-Garrado, J. Ignés-Mullol, J. Claret, and F. Sagués, Phys. Rev. Lett. 103, 237802 (2009).

[14] R. Fasel, M. Parschau, and K.-H. Ernst, Nature, 439, 449 (2006).

[15] M. Parschau, S. Romer, and K.-H. Ernst, J. Am. Chem. Soc., 126, 15398 (2004).

[16] N. Katsonis, H. Xu, R. M. Haak, T. Kubernac, Ž. Tomović, S. George, M. Van der Auweraer, A. P. H. J. Shenning, E. W. Meijer, B. L. Feringa, and S. De Feyter, Angew. Chem. Int. Ed., 47, 4997 (2008).

[17] A. M. Berg, D. L. Patrick, Angew. Chem. Int. Ed., 44, 1821 (2005). 